\title{
Improved imaging of colorectal liver metastases using single-source, fast kVp-switching, dual-energy CT: preliminary results
}

\author{
Przemysław Ratajczak ${ }^{1 A, B, D, E}$, Zbigniew Serafin ${ }^{1 A, C, D, E, F}$, Agata Sławińska ${ }^{1 B, F,}$, Maciej Słupski2A,B,D, \\ Waldemar Leszczyński ${ }^{1 C, D}$
}

'Department of Radiology and Diagnostic Imaging, Nicolaus Copernicus University, Collegium Medicum, Bydgoszcz, Poland 2Department of Hepatobiliary and General Surgery, Nicolaus Copernicus University, Collegium Medicum, Bydgoszcz, Poland

\section{Abstract}

\begin{abstract}
Purpose: Computed tomography remains the first-choice modality for assessment of colorectal cancer liver metastases (CRLM). Dual-energy computed tomography (DECT) is a relatively new technique that is becoming increasingly available. One of the advantages of DECT is the ability to maximise iodine detection. Our aim was to test whether single-source, fast kVp-switching DECT can improve imaging quality of CRLM compared to conventional (polychromatic) CT.
\end{abstract}

Material and methods: Twenty consecutive patients were enrolled into a preliminary prospective study. The scanning protocol consisted of four phases: non-contrast with standard $120 \mathrm{kV}$ tube voltage and three post-contrast phases with rapid voltage switching. As a result, three sets of images were reconstructed: pre- and postcontrast polychromatic (PR), monochromatic (MR), and iodine concentration map (IM). To compare the sensitivity of the tested reconstructions, the number of CRLMs and the maximum diameter of the largest lesion were calculated. Objective image quality was measured as signal-to-noise ratio (SNR) and contrast-to-noise ratio (CNR). The radiation dose was expressed as CTDI ${ }_{\text {vol }}$.

Results: Imaging was successfully performed in all patients. The number of detected lesions was significantly lower on PR images than on IM and MR 50-70 keV (mean number: 4.20 and 4.45, respectively). IM and MR at $70 \mathrm{keV}$ presented the highest quality. SNR was significantly higher for IM and $70 \mathrm{keV}$ images than for other reconstructions. The mean radiation dose was $14.61 \mathrm{mGy}$ for non-contrast $120 \mathrm{kV}$ scan and $17.89 \mathrm{mGy}$ for single DECT scan $(p<0.05)$.

Conclusions: DECT is a promising tool for CRLM imaging. IM and low-photon energy MR present the highest differences in contrast between metastases and the normal liver parenchyma.

Key words: liver metastases, rectal cancer, dual-energy computed tomography, oncology, staging.

\section{Introduction}

Colorectal cancer (CRC) is the third most common malignancy and the fourth leading cause of cancer-related death worldwide [1]. Each year, 1.36 million new cases of CRC are diagnosed, affecting 17.2 per 100,000 people. Approximately $25 \%$ of patients present with metastases at the time of diagnosis, with the liver being most commonly affected by metastatic lesions. Moreover, almost a half of patients with CRC will develop metastases during treatment [2]. Since both the location and number of metastases are crucial for surgical resection, preoperative liver imaging is of great importance [3-6]. Detailed presentation of lesions is important when precisely targeted methods of treatment are intended to be used, including laparoscopy, percutaneous ablation, chemoembolisation, and radioembolisation [7-9].

\section{Address for correspondence:}

Prof. Zbigniew Serafin, Department of Radiology and Diagnostic Imaging, Nicolaus Copernicus University, Collegium Medicum, 9 M. Skłodowskiej-Curie St.,

85-094 Bydgoszcz, Poland, e-mail: serafin@cm.umk.pl

Authors' contribution:

A Study design · B Data collection · C Statistical analysis · D Data interpretation · E Manuscript preparation · F Literature search · G Funds collection 
Currently, contrast-enhanced computed tomography (CT) and magnetic resonance imaging (MRI) are the most commonly used modalities for preoperative imaging of colorectal liver metastasis (CRLM), with sensitivity rates of $70-86 \%$ and specificity rates of $93-95 \%[5,6]$. Newer MRI techniques, including MR elastography, are still a work in progress [10]. Although conventional ultrasonography (US) is of limited value for detecting CRLM [11], recent studies suggest that contrast-enhanced US (CEUS), intraoperative US (IOUS), and intraoperative contrast-enhanced US (CEIOUS) may have better performance, with sensitivity and specificity of up to $94 \%$ and $77 \%$, respectively [12-14]. Fluorodeoxyglucose positron emission tomography (FDG-PET) and FDG-PET/CT are usually performed only in patients after adjuvant or neoadjuvant chemotherapy. However, the sensitivity and specificity of FDG-PET/CT in naive patients are as high as 97\% [5].

Despite its limited diagnostic performance, CT remains the first-choice modality for liver metastases screening in patients with CRC, preoperative estimation of the remnant liver size, and defining vascular anatomy [15]. Dual-energy CT (DECT) is a promising technique that can increase the diagnostic accuracy of CT in detecting CRLM because it allows material decomposition [16] and mapping of iodine uptake in hepatic lesions [17]. Moreover, DECT has been shown to improve image contrast in suboptimal contrast conditions [18]. Since CRLMs are usually hypovascular, dual-energy techniques may significantly increase the sensitivity of CT in detecting these lesions.

In this study, we aimed to test whether single-source fast $\mathrm{kVp}$-switching DECT can improve imaging quality of colorectal liver metastases compared to conventional polychromatic CT.

\section{Material and methods}

\section{Patients}

A total of 20 consecutive patients ( 11 men and nine women, aged 33-79 years [mean age 62 years] were enrolled

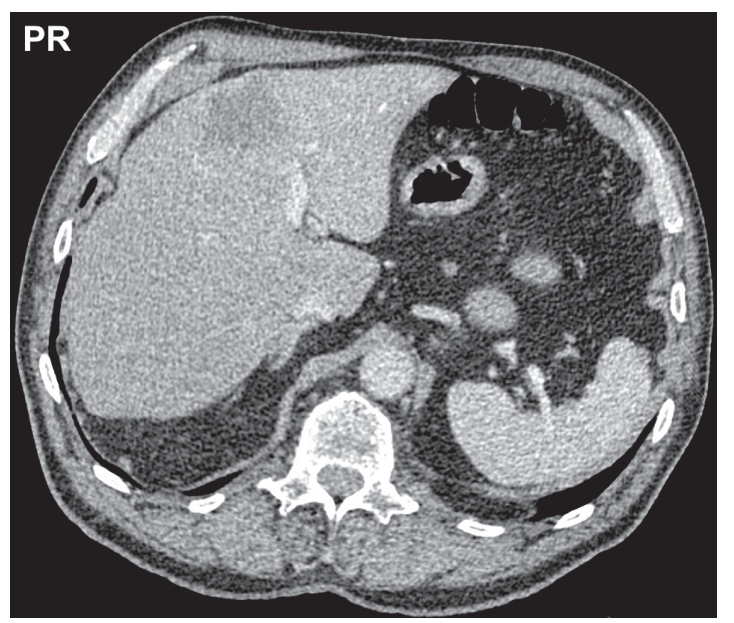

Figure 1. A large metastasis in segment 4a in polychromatic reconstruction between January and July 2016 into this preliminary prospective study. We included patients who were scheduled for partial hepatectomy due to CRLM. Exclusion criteria were as follows: age below 18 years, pregnancy or lactation, metallic implants in the abdomen, and contraindications to iodinated contrast media. Informed consent was obtained from all participants, and the study was approved by our University Review Board.

\section{DECT scanning protocol}

Examinations were performed with a Discovery CT 750HD scanner (GE Healthcare, Waukesha, USA) using a dual-energy spectral CT in a GEM technology (Gemstone Spectral Imaging). It is a DECT technique based on rapid switching between high- and low-energy photon emission from a single source [19]. Tube voltage switching between 80 and $140 \mathrm{kV}$ enabled reconstruction of polychromatic (conventional) images at peak $140 \mathrm{kVp}$, virtual monochromatic images at photon energies ranging from 40 to $140 \mathrm{keV}$, and calculation of tissue iodine concentration maps.

A four-phase CT protocol was performed, which consisted of a non-enhanced phase (NP), arterial phase (AP), portal venous phase (PP), and venous phase (delayed phase, VP). The scan range was from the dome of the liver to the iliac crest during an inspiration. NP scans were acquired with a tube voltage of $120 \mathrm{kVp}$, detector collimation of $0.625 \times 64 \mathrm{~mm}$, rotation speed of $0.6 \mathrm{~s}$, and helical pitch of $1.375: 1$. Then, a non-ionic contrast medium (Ultravist 300, Bayer Pharma AG, Germany) was administered intravenously with a power injector at $3 \mathrm{ml} / \mathrm{s}$ (total dose of $1 \mathrm{ml} / \mathrm{kg}$ ) followed by a $20-\mathrm{ml}$ bolus of $0.9 \% \mathrm{NaCl}$ at $3 \mathrm{ml} / \mathrm{s}$. Delay times for each of the phases were as follows: AP - $30 \mathrm{~s}, \mathrm{PP}-60 \mathrm{~s}$, and VP - $180 \mathrm{~s}$ after contrast agent injection. Contrast-enhanced phases were acquired in the GSI scanning mode using the following scan parameters: tube voltage switching between 80 and $140 \mathrm{kVp}$ within $0.5 \mathrm{~ms}$, detector collimation of $0.625 \times 64 \mathrm{~mm}$, rotation speed of 0.6-0.8 s, and helical pitch of 1.375:1. ASIR (Adaptive Statistical Iterative Reconstruction) option was disabled.

\section{Image reconstruction and analysis}

Post-processing was performed on a dedicated workstation (AW4.6, GE Healthcare, Waukesha, USA). The reconstructed slice thickness was $1.25 \mathrm{~mm}$. Three types of images were generated, including a classic polychromatic reconstruction at $140 \mathrm{kVp}(\mathrm{PR}$, Figure 1), monochromatic virtual reconstruction $(\mathrm{MR})$ at photon energies ranging from $40 \mathrm{keV}$ to $140 \mathrm{keV}$ (10-keV interval, 11 sets of images), and iodine over water maps (IM, Figure 2). In total, 13 sets of axial images were evaluated for each patient.

Images were assessed by two radiologists (each with five years of experience in abdominal CT) [10]. Subjective 


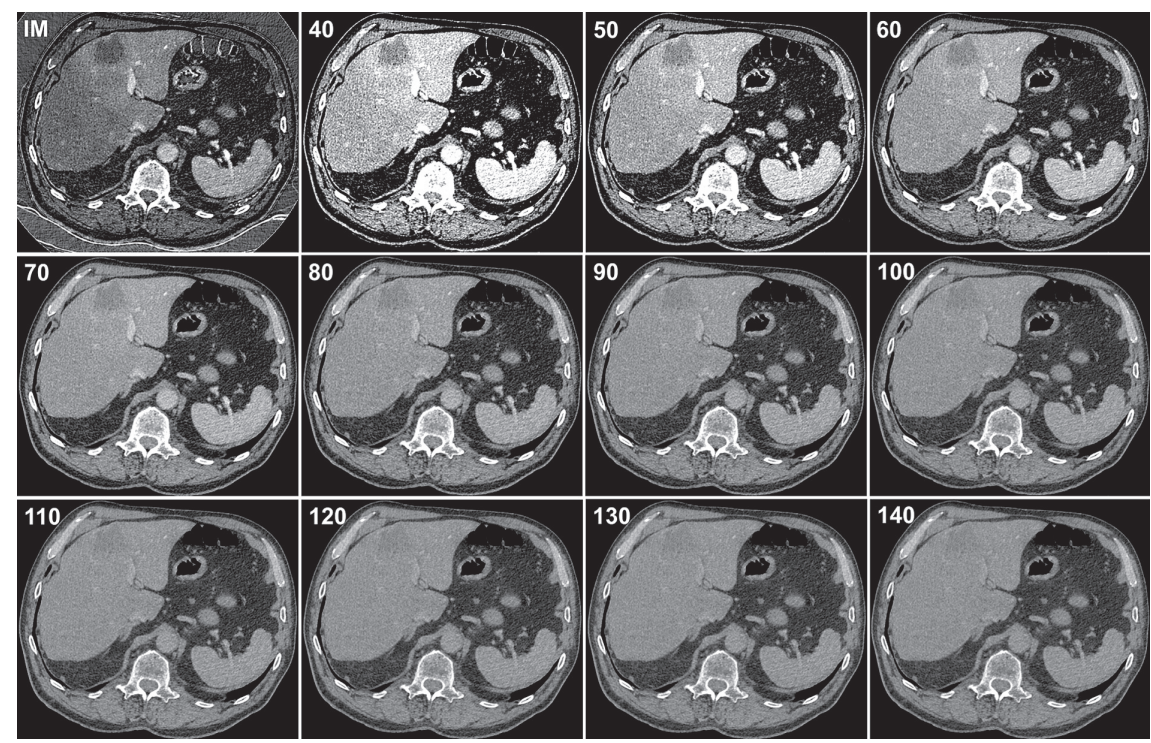

Figure 2. The metastasis presented in Figure 1 in iodine map (IM) and monoenergetic reconstructions at 40-140 keV

image quality of the conspicuity of metastases was rated as follows: 1 - excellent, 2 - good, 3 - poor, 4 - invisible. Objective image quality was measured as signal-to-noise ratio (SNR) and contrast-to-noise ratio (CNR). The ratios were calculated based on the portal phase of contrastenhanced images using attenuation standard deviation of visceral adipose tissue as the noise value (N), CT number of the major lesion as the signal value (S), and the difference in CT numbers between the normal liver parenchyma and the major lesion as the contrast value (C). To compare the sensitivities of the tested reconstructions, the number of CRLMs and the largest diameter of the largest lesion were determined.

The appearance of CRLM was compared between different reconstructions based on CT numbers of the largest lesion. In the first step, the lesion was identified on polychromatic portal phase images, and a circular region of interest (ROI) was placed to cover the largest possible cross-sectional area of the metastasis. Then, the ROI was propagated to all other phases and reconstructions corrected manually if necessary. CT numbers were measured on polychromatic and monochromatic images. Iodine concentration $\left(\mu \mathrm{g} / \mathrm{cm}^{3}\right)$ was measured on iodine maps.

\section{Statistical analysis}

Parametric data were expressed as mean values with 95\% confidence intervals (95\% CI). Radiation dose was expressed as $\mathrm{CTDI}_{\mathrm{vol}}$. The reference diameter and the number of lesions were assessed on PR images. The normality of data was assessed using the Kolmogorov-Smirnov test. Repeated measures analysis of variance (ANOVA) was used to test for potential differences between reconstructions in the subjective image quality, the number of lesions, and the diameter of the largest metastasis. Differences between reconstructions were tested using the Friedman test or the repeated measures ANOVA test.
The intraclass correlation coefficient was used to test interobserver agreement with respect to the number of lesions and the size of the largest lesion. A $p$ value $<0.05$ was considered significant. Statistical analyses were performed using Statistica 10 (StatSoft Inc., Tulsa, OK) and MedCalc Statistical Software version 13.3 (MedCalc Software bvba, Ostend, Belgium).

\section{Results}

Imaging was successfully performed in all patients, and no adverse reactions were observed. None of the examinations was rejected due to inadequate image quality. The mean radiation dose was $14.61 \mathrm{mGy}$ for $\mathrm{NP}$ and $17.89 \mathrm{mGy}$ for DECT phases, respectively $(p<0.05)$.

The results of the image quality analysis are presented in Table 1. The highest subjective image quality was rated for monoenergetic reconstructions at 120,130, and $140 \mathrm{keV}(p<0.0001)$. The diameter of metastases ranged between 12 and $193 \mathrm{~mm}$ (mean diameter: 58.4, 95\% CI: 34.3-82.4 mm). The Friedman test revealed significant differences between reconstructions $(p=0.0070)$, but in post-hoc analysis there was no discrepancy between PR and monoenergetic images. The number of lesions varied between 1 and $20 \mathrm{~mm}$ (mean size: 4.20, 95\% CI: 1.37-7.03 $\mathrm{mm}$ ); the Friedman test revealed significant differences between reconstructions ( $p=0.0495)$. The post-hoc analysis showed that the number of lesions was significantly lower on PR images than on IM and on monoenergetic reconstructions at 50,60, and $70 \mathrm{keV}$ (mean number: 4.20 and 4.45 , respectively). As regards the objective assessment, IM and monoenergetic reconstruction at $70 \mathrm{keV}$ presented the highest quality. SNR was significantly higher for IM and $70 \mathrm{keV}$ images than for other reconstructions. Differences in CNR were less pronounced, as the only significant difference was observed between $70 \mathrm{keV}$ images and polychromatic reconstructions. 
Table 1. Image quality parameters for the assessed reconstructions. Mean values and 95\% Cls are given

\begin{tabular}{|l|c|c|c|c|c|} 
& Image quality score & No. of lesions & Largest lesion diameter $(\mathrm{mm})$ & SNR & CNR \\
\hline IM & $2.80(2.51-3.09)$ & $4.45(1.56-7.36)$ & $58.6(34.6-82.6)$ & $3.40(2.63-4.20)$ & $2.06(1.37-2.55)$ \\
\hline PR & $2.10(1.70-2.50)$ & $4.20(1.37-7.03)$ & $58.2(34.0-82.5)$ & $2.48(1.95-3.02)$ & $1.34(0.91-1.77)$ \\
\hline MR40 & $1.95(1.59-2.31)$ & $4.10(1.27-6.93)$ & $57.3(33.3-81.3)$ & $2.91(2.24-3.58)$ & $1.99(1.28-2.69)$ \\
\hline MR50 & $1.50(1.26-1.74)$ & $4.45(1.55-7.35)$ & $58.5(34.5-82.6)$ & $2.91(2.25-3.57)$ & $1.86(1.20-2.52)$ \\
\hline MR60 & $1.55(1.23-1.87)$ & $4.45(1.55-7.35)$ & $58.1(34.2-82.0)$ & $3.06(2.32-3.81)$ & $2.08(1.38-2.78)$ \\
\hline MR70 & $1.70(1.36-2.04)$ & $4.45(1.55-7.35)$ & $58.3(34.5-82.2)$ & $3.41(2.60-4.23)$ & $1.91(1.27-2.54)$ \\
\hline MR80 & $2.10(1.70-2.50)$ & $4.35(1.50-7.20)$ & $58.5(34.5-82.4)$ & $2.83(2.16-3.50)$ & $1.51(1.04-1.98)$ \\
\hline MR90 & $2.20(1.84-2.56)$ & $4.35(1.50-7.20)$ & $58.6(34.7-82.5)$ & $2.72(2.03-3.41)$ & $1.42(0.99-1.86)$ \\
\hline MR100 & $2.35(1.97-2.73)$ & $4.30(1.45-7.15)$ & $58.2(34.3-82.2)$ & $2.63(1.94-3.32)$ & $1.33(0.92-1.74)$ \\
\hline MR110 & $2.40(2.02-2.78)$ & $4.25(1.39-7.11)$ & $57.6(33.5-81.7)$ & $2.57(1.88-3.26)$ & $1.25(0.86-1.65)$ \\
\hline MR120 & $2.65(2.34-2.96)$ & $4.30(1.44-7.16)$ & $58.0(34.0-82.1)$ & $2.51(1.82-3.19)$ & $1.43(0.98-1.88)$ \\
\hline MR130 & $2.80(2.51-3.09)$ & $4.25(1.43-7.07)$ & $57.9(33.8-82.1)$ & $2.47(1.79-3.14)$ & $1.14(0.76-1.53)$ \\
\hline MR140 & $2.80(2.51-3.09)$ & $4.15(1.31-6.99)$ & $57.4(33.3-81.5)$ & $2.27(1.54-3.01)$ & $1.25(0.56-1.94)$ \\
\hline
\end{tabular}

SNR - signal-to-noise ratio, CNR - contrast-to-noise ratio, IM - iodine maps, PR - polychromatic reconstructions, MR - monochromatic reconstructions

Table 2. Mean attenuation values at arterial, portal, and venous phases of contrast enhancement of the normal liver parenchyma and the metastatic lesions as assessed by different reconstructions

\begin{tabular}{|l|c|c|c|c|c|c|}
\hline \multirow{2}{*}{} & \multicolumn{3}{|c|}{ Normal liver } & \multicolumn{3}{c|}{ Metastases } \\
\cline { 2 - 7 } & Arterial phase & Portal phase & Venous phase & Arterial phase & Portal phase & Venous phase \\
\hline IM & 6.0 & 21.7 & 13.1 & 8.0 & 16.3 & 13.2 \\
\hline PR & 59.8 & 83.4 & 71.3 & 8.0 & 16.3 & 13.2 \\
\hline MR40 & 98.1 & 212.9 & 149.9 & 49.8 & 62.0 & 59.9 \\
\hline MR60 & 79.2 & 155.0 & 111.8 & 97.5 & 160.0 & 139.3 \\
\hline MR70 & 67.2 & 116.3 & 88.0 & 74.0 & 112.8 & 100.5 \\
\hline MR80 & 60.1 & 92.2 & 73.4 & 60.8 & 83.4 & 76.8 \\
\hline MR90 & 55.7 & 76.8 & 62.9 & 50.9 & 66.1 & 62.0 \\
\hline MR100 & 52.7 & 67.4 & 58.3 & 44.5 & 54.9 & 52.9 \\
\hline MR110 & 40.6 & 59.8 & 54.0 & 40.9 & 46.5 & 46.0 \\
\hline MR120 & 48.1 & 54.8 & 50.8 & 38.3 & 41.2 & 42.2 \\
\hline MR130 & 47.2 & 51.3 & 48.9 & 37.7 & 38.0 & 39.1 \\
\hline MR140 & 46.6 & 46.6 & 47.2 & 35.1 & 34.8 & 37.0 \\
\hline
\end{tabular}

IM - iodine maps, PR - polychromatic reconstructions, MR - monochromatic reconstructions

Attenuation values of the normal liver parenchyma and those of metastases are presented in Table 2 and Figure 3. These values were statistically different between the tested reconstructions $(p<0.001)$. The relative difference in attenuation (or iodine content) between the liver parenchyma and the largest lesion is presented in Figure 4. In the arterial phase, the difference was the highest for IM (mean 58\%, 95\% CI: 32-83\%). There was a significant within-reconstruction effect in ANOVA ( $p=0.005)$, and IM values were significantly higher than PR values ( $p=0.0488$ ). The highest difference in the portal phase was observed for IM (mean 51\%, 95\% CI: 35-68\%) and in the venous phase for $140 \mathrm{keV}$ (mean 33\%, 95\% CI: 20-47\%), with no significant within-reconstruction effects (Figure 5).
The interobserver agreement for the number of lesions was high. It was the lowest for PR (ICC 0.95, 95\% CI: 0.88-0.98), followed by monoenergetic reconstructions at $40 \mathrm{keV}$ (ICC 0.96, 95\% CI: 0.90-0.99). The remaining reconstructions presented an ICC of 0.99 . The interobserver agreement for the diameter of the largest lesion was 0.99 (0.99-1.00) for all reconstructions.

\section{Discussion}

To our knowledge, this is the first study to analyse the technical quality of single-source, fast $\mathrm{kVp}$-switching DECT in the imaging of CRLM. The main finding of our study is that some monoenergetic reconstructions may re- 

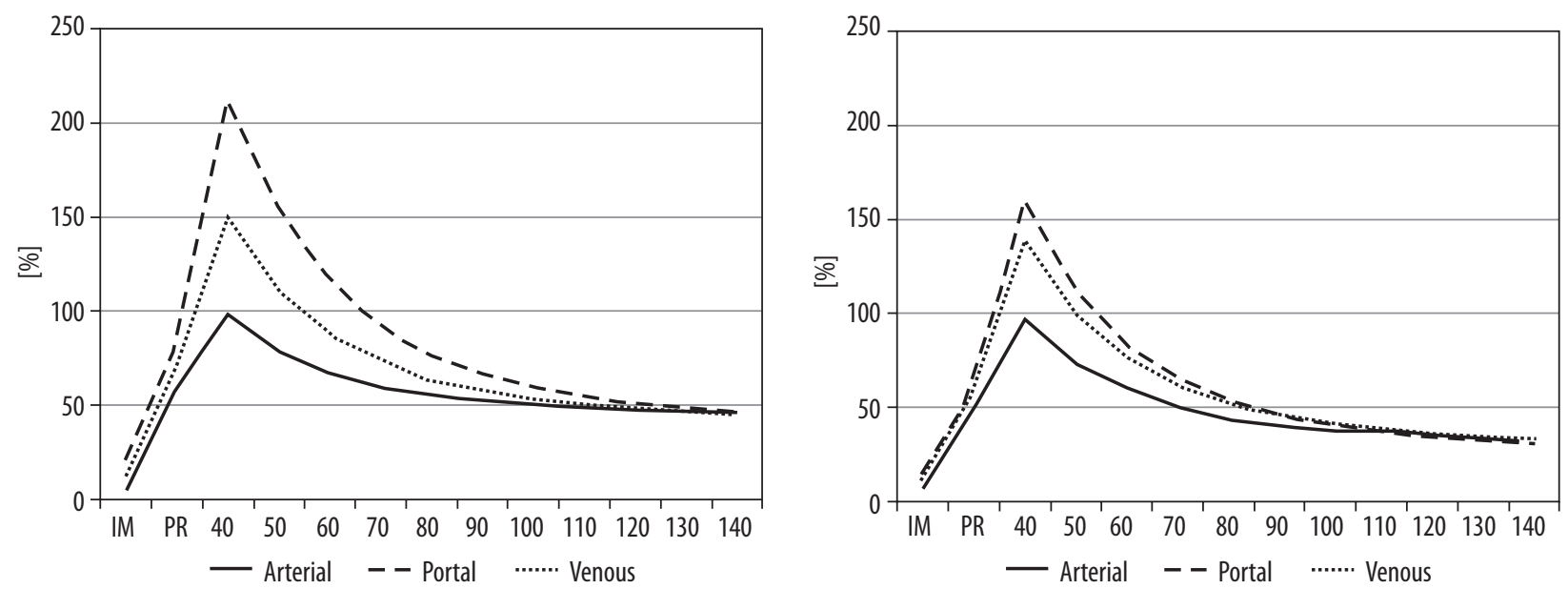

Figure 3. Signal intensity (HU equivalent) of the normal liver parenchyma (A) and the largest metastasis (B) in arterial, portal, and venous phases of contrast enhancement for the tested image reconstructions (IM - iodine maps, PR - polychromatic reconstruction, and monoenergetic reconstructions at 40-140 keV)

veal more metastases than polychromatic reconstructions. Moreover, iodine maps showed a higher signal difference between lesions and the normal liver parenchyma than did conventional CT images.

DECT may be used to improve the image quality of CT of the liver. Recently, Sudarski et al. compared objective and subjective image quality of PR and MR for GIST liver metastases. They found that less experienced readers might improve their diagnostic confidence in evaluating metastases by using $70 \mathrm{keV}$ MR instead of conventional PR, because MR images at $70 \mathrm{keV}$ were given the highest subjective quality ratings regardless of patient size [20]. In our study, the highest subjective image quality was rated for reconstructions at 120,130, and $140 \mathrm{keV}$, but images at $60 \mathrm{keV}$ and $70 \mathrm{keV}$ presented the highest values of SNR and CNR among MRs. However, in view of the study by Nattenmüller et al., the added value of DECT for the imaging of the liver is not clear [21]. Those authors tried to verify the role of DECT in depicting hypodense liver metastases within steatotic

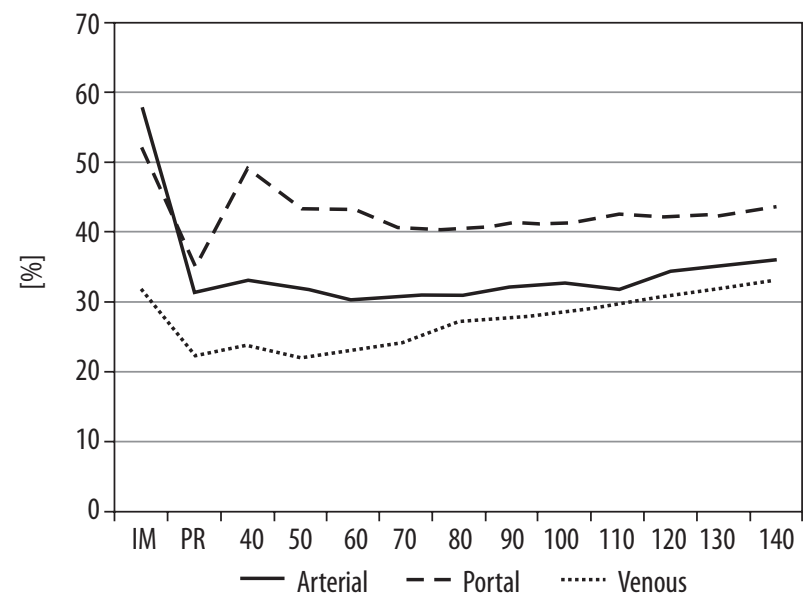

Figure 4. Relative difference in the signal intensity (HU equivalent) of the normal liver parenchyma and the largest metastasis in arterial, portal, and venous phases of contrast enhancement for the tested image reconstructions (IM - iodine maps, PR - polychromatic reconstruction, and monoenergetic reconstructions at 40-140 keV) liver parenchyma. Although they found that MR images at $120 \mathrm{kVp}$ had the best quantitative and qualitative parameters, they concluded that additional radiation exposure due to dual-energy acquisition is not clinically justified. On the other hand, our results indicate that DECT is characterised by improved lesion detection on MRs, which is a significant practical advantage. Similar results were presented by Altenbernd et al., who evaluated CRLM using just two MRs ( $80 \mathrm{keV}$ and $120 \mathrm{keV}$ ) with nine different window settings [22]. They concluded that $80 \mathrm{kVp}$ images were more precise in detecting liver metastases than $120 \mathrm{kVp}$ images and that window settings had a significant influence on conspicuity. Specifically, MR at $80 \mathrm{keV}$ and the 50/350 HU window setting revealed a significantly higher total number of metastases and a higher number of small metastases $(<1 \mathrm{~mm}$ in size) than $120 \mathrm{keV}$ reconstructions.

As shown in Figure 3, signal intensity of the normal liver parenchyma and metastases is not distributed uniformly, with higher CT values in low-energy reconstruc-

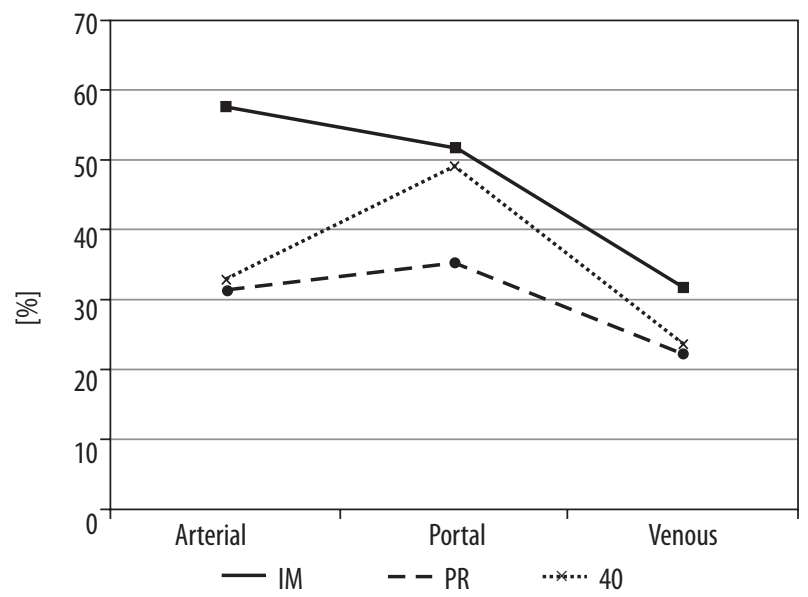

Figure 5. Relative difference in the signal intensity (HU equivalent) of the normal liver parenchyma and the largest metastasis in arterial, portal, and venous phases of contrast enhancement for iodine maps (IM), polychromatic reconstruction (PR), and monoenergetic reconstruction at $40 \mathrm{keV}$ 
tions. Similar spectral curves were presented by Wang et al. [23]. They also concluded that the best presentation of liver tumours is achieved in the portal venous phase because of improved contrast agent dispersion in comparison to the arterial phase. According to our results, this observation is accurate for both monoenergetic and polyenergetic reconstructions. However, on iodine maps, the difference in attenuation between the normal liver parenchyma and CRLM is highest in the arterial phase (Figure 5). This may be explained by the unique sensitivity of IM in detecting the content of contrast medium in the tissue.

The difference in the signal between structures is the most important factor that allows for lesion identification. In an early work by Robinson et al., pure $80 \mathrm{kVp}$ images acquired using a dual-source dual-energy scanner demonstrated higher attenuation differences between hepatic metastases and the normal liver parenchyma than $120 \mathrm{kVp}$ images [24]. In our study, the highest differences between the liver parenchyma and metastases were noted on iodine maps, $40 \mathrm{keV}$ images, and $140 \mathrm{keV}$ images (Figure 4). Since IM has also superior objective image quality, it seems to be the best reconstruction mode for diagnosing CRLM. Interestingly, IM was the only image type in which the arterial phase was more sensitive in detecting metastases than the portal phase. This may be explained by a unique sensitivity of IM to the content of iodine in the tissues. One might thus ask the question of whether the arterial phase can also be sufficient for diagnosis, which would significantly reduce radiation exposure. The above-described technical advantages of IM and monoenergetic reconstructions are in line with the most important result of our study, namely that DECT can reveal more metastases than conventional CT, because the precise determination of the cancer dissemination is essential for surgery planning.

The study has some limitations that have to be addressed. First, because the study was preliminary, a small number of participants were included. Although we found some advantages of DECT in imaging for CRLM, these findings should to be confirmed in a larger sample. Second, only one contrast medium at a low concentration was tested. Because iodine maps most effectively depict the differences in enhancement between the normal parenchyma and metastases, it would be valuable to investigate the performance of contrast media at higher concentrations. Third, no dose reduction strategies were applied in the study. Potentially, iterative reconstruction would have allowed for dose reduction to a level close to that associated with conventional CT. However, the aim of our study was to test DECT reconstructions, and the effect of dose reduction may be investigated in another study. Finally, there was no objective reference method established to objectively measure the diagnostic value of DECT for the determination of hepatic CRC involvement. Therefore, further studies are being conducted with IOUS as a reference method to precisely measure the diagnostic performance of DECT in revealing CRLM.

\section{Conclusions}

DECT seems to be a promising tool for imaging of CRLM. Iodine maps and low-photon energy reconstructions present the highest differences in contrast between metastases and the normal liver parenchyma. Larger studies are necessary to establish the role of DECT in determining liver involvement in colorectal cancer.

\section{Disclosure}

Some of the data from this study were presented at the $41^{\text {st }}$ Congress of the Polish Medical Society of Radiology, Kraków, 2016.

\section{Conflict of interest}

The authors report no conflict of interest.

\section{References}

1. Ferlay J, Soerjomataram I, Dikshit R, et al. Cancer incidence and mortality worldwide: sources, methods and major patterns in GLOBOCAN 2012. Int J Cancer 2015; 136: E359-386.

2. Van Cutsem E, Cervante A, Nordlinger B, et al. Metastatic colorectal cancer: ESMO Clinical Practice Guidelines for diagnosis, treatment and follow-up. Ann Oncol 2014; 25: iiil-9.

3. Jones RP, Vauthey JN, Adam R, et al. Effect of specialist decision-making on treatment strategies for colorectal liver metastases. Br J Surg 2012; 99: 1263-1269.

4. Siriwardena AK, Mason JM, Mullamitha S, et al. Management of colorectal cancer presenting with synchronous liver metastases. Nat Rev Clin Oncol 2014; 11: 446-459.

5. Niekel MC, Bipat S, Stoker J. Diagnostic imaging of colorectal liver metastases with CT, MR imaging, FDG PET, and/or FDG PET/CT: a meta-analysis of prospective studies including patients who have not previously undergone treatment. Radiology 2010; 257: 674-684.

6. van Kessel CS, Buckens CF, van den Bosch MA, et al. Preoperative imaging of colorectal liver metastases after neoadjuvant chemotherapy: a meta-analysis. Ann Surg Oncol 2012; 19: 2805-2813.

7. Cheng Y, Zhang L, Li H, et al. Laparoscopic versus open liver resection for colorectal liver metastases: a systematic review. J Surg Res 2017; 220: 234-246.

8. Venkat SR, Mohan PP, Gandhi RT. Colorectal Liver Metastasis: Overview of Treatment Paradigm Highlighting the Role of Ablation. AJR Am J Roentgenol 2018; 210: 883-890.

9. Fairchild AH, White SB. Decision Making in Interventional Oncology: Intra-arterial Therapies for Metastatic Colorectal Cancer-Y90 and Chemoembolization. Semin Intervent Radiol 2017; 34: 87-91. 
10. Rusak G, Zawada E, Lemanowicz A, et al. Whole-organ and segmental stiffness measured with liver magnetic resonance elastography in healthy adults: significance of the region of interest. Abdom Imaging 2015; 40: 776-782.

11. Larsen LP, Rosenkilde M, Christensen H, et al. The value of contrast enhanced ultrasonography in detection of liver metastases from colorectal cancer: a prospective double-blinded study. Eur J Radiol 2007; 62: 302-307.

12. Strobel D, Bernatik T, Blank W, et al. Diagnostic accuracy of CEUS in the differential diagnosis of small $(</=20 \mathrm{~mm})$ and subcentimetric $(</=10 \mathrm{~mm})$ focal liver lesions in comparison with histology. Results of the DEGUM multicenter trial. Ultraschall Med 2011; 32: 593-597.

13. Lordan JT, Stenson KM, Karanjia ND. The value of intraoperative ultrasound and preoperative imaging, individually and in combination, in liver resection for metastatic colorectal cancer. Ann R Coll Surg Engl 2011; 93: 246-249.

14. Schulz A, Dormagen JB, Drolsum A, et al. Impact of contrast-enhanced intraoperative ultrasound on operation strategy in case of colorectal liver metastasis. Acta Radiol 2012; 53: 1081-1087.

15. Frankel TL, Gian RK, Jarnagin WR. Preoperative imaging for hepatic resection of colorectal cancer metastasis. J Gastrointest Oncol 2012; 3: 11-18.

16. Chai Y, Xing J, Gao J, et al. Feasibility of virtual nonenhanced images derived from single-source fast $\mathrm{kVp}$-switching dual-energy $\mathrm{CT}$ in evaluating gastric tumors. Eur J Radiol 2016; 85: 366-372.

17. Nattenmüller J, Hosch W, Nguyen TT, et al. Hypodense liver lesions in patients with hepatic steatosis: do we profit from dual-energy computed tomography? Eur Radiol 2015; 25: 3567-3576.
18. Schabel C, Bongers M, Sedlmair M, et al. Assessment of the hepatic veins in poor contrast conditions using dual energy CT: evaluation of a novel monoenergetic extrapolation software algorithm. Rofo 2014; 186: 591-597.

19. Zhang D, Li X, Liu B. Objective characterization of GE discovery CT750 HD scanner: gemstone spectral imaging mode. Med Phys 2011; 38: 1178-1188.

20. Sudarski S, Apfaltrer P, Nance JW Jr, et al. Objective and subjective image quality of liver parenchyma and hepatic metastases with virtual monoenergetic dual-source dual-energy CT reconstructions: an analysis in patients with gastrointestinal stromal tumor. Acad Radiol 2014; 21: 514-522.

21. Nattenmüller J, Hosch W, Nguyen TT, et al. Hypodense liver lesions in patients with hepatic steatosis: do we profit from dual-energy computed tomography? Eur Radiol 2015; 25: 3567-3576.

22. Altenbernd J, Forsting M, Lauenstein T, et al. Improved image quality and detectability of hypovascular liver metastases on DECT with different adjusted window settings. Rofo 2017; 189: 228-232.

23. Wang Q, Shi G, Qi X, et al. Quantitative analysis of the dual-energy CT virtual spectral curve for focal liver lesions characterization. Eur J Radiol 2014; 83: 1759-1764.

24. Robinson E, Babb J, Chandarana H, et al. Dual source dual energy MDCT: comparison of $80 \mathrm{kVp}$ and weighted average $120 \mathrm{kVp}$ data for conspicuity of hypo-vascular liver metastases. Invest Radiol 2010; 45: 413-418. 